This is an Accepted Manuscript of an article published by Taylor \& Francis in Drug Development and Industrial Pharmacy on 25/09/18, available online: https://www.tandfonline.com/doi/ abs/10.1080/03639045.2018.1503298?journalCode=iddi20 


\title{
Preparation \& Optimization of Monodisperse Polymeric Microparticles Using Modified Vibrating Orifice Aerosol Generator for Controlled Delivery of Letrozole in Breast Cancer Therapy
}

\author{
Bayan Alemrayat ${ }^{1}$, Mohamed A. Elrayess ${ }^{2}$, Raid G. Alany ${ }^{3}$, Abdelbary Elhissi ${ }^{1,4}$, Husam
} M. Younes ${ }^{1,4^{*}}$

\footnotetext{
${ }^{1}$ Pharmaceutics and Polymeric Drug Delivery Research Laboratory, College of Pharmacy, Qatar University, P.O. Box 2713, Doha, Qatar

${ }^{2}$ Anti-Doping Lab Qatar, Doha, Qatar

${ }^{3}$ Drug Discovery, Delivery and Patient Care Theme, School of Life Sciences, Pharmacy and Chemistry, Kingston University London, United Kingdom

${ }^{4}$ Office of Vice President for Research and Graduate Studies, Qatar University, P.O. Box 2713, Doha, Qatar
}

\author{
*Corresponding author: \\ Dr. Husam Younes \\ Pharmaceutics \& Polymeric Drug Delivery Research Laboratory \\ College of Pharmacy, Qatar University, P.O. Box 2713 \\ Doha, Qatar, and \\ Office of the Vice President for Research and Graduate Studies \\ Qatar University, P.O. Box 2713 \\ Doha, Qatar \\ E: husamy@qu.edu.qa \\ $\mathrm{T}:+974$ 4403-5583 \\ F: +974 4403-390
}




\begin{abstract}
Letrozole (LTZ) is effective for the treatment of hormone-receptor-positive breast cancer in postmenopausal women. In this work, and for the first time, using Vibrating Orifice Aerosol Generator (VOAG) technology, monodisperse poly- $\varepsilon$-caprolactone (PCL) and poly (D, L-Lactide) (PDLLA) LTZ-loaded microparticles were prepared and found to elicit selective high cytotoxicity against cancerous breast cells with no apparent toxicity on healthy cells in vitro. Plackett-Burman experimental design was utilized to identify the most significant factors affecting particle size distribution to optimize the prepared particles. The generated microparticles were characterized in terms of microscopic morphology, size, drug entrapment efficiency and release profile over one-month period. Long-term cytotoxicity of the microparticles was also investigated using MCF-7 human breast cancer cell lines in comparison with primary mammary epithelial cells (MEC). The prepared polymeric particles were monodispersed, spherical and apparently smooth, regardless of the polymer used or the loaded LTZ concentration. Particle size varied from $15.6 \mu \mathrm{m}$ to $91.6 \mu \mathrm{m}$ and from $22.7 \mu \mathrm{m}$ to $99.6 \mu \mathrm{m}$ with size distribution (expressed as span values) ranging from 0.22 to 1.24 and from 0.29 to 1.48 for PCL and PDLLA based microparticles, respectively. Upon optimizing the manufacture parameters, span was reduced to $0.162-0.195$. Drug entrapment reached as high as $96.8 \%$, and drug release from PDLLA and PCL followed a biphasic zero-order release using $5 \%$ or $30 \% \mathrm{w} / \mathrm{w}$ drug loading in the formulations. Long term in vitro cytotoxicity studies indicated that microparticles formulations significantly inhibited the growth of MCF-7 cell line over a prolonged period of time but did not have toxic effects on the normal breast epithelial cells.
\end{abstract}

Keywords: vibrating orifice aerosol generator, controlled release, letrozole, monodisperse, microparticles, size distribution, cancer. 


\section{Introduction}

Letrozole (LTZ) is an FDA approved adjuvant therapy for the treatment of estrogen receptor (ER)-positive breast cancer in postmenopausal women [1]. Clinical trials have proven that LTZ can increase survival in breast cancer victims and reduce risk of recurrence and metastasis $[2,3]$. Marketed oral formulations of LTZ may provide a non-linear release [4], resulting in plasma levels above the therapeutic window with concomitant severe adverse effects such as arthralgia, bone fractures, nausea, fatigue, and thromboembolic events [5]. Thus, it is highly desirable to design new delivery systems of LTZ with more predictable pharmacokinetic profiles in order to keep the plasma drug concentration below the toxic levels.

Polymeric delivery systems may provide an approach to enhance solubility, prolong halflife and reduce toxicity of many therapeutic molecules. Moreover, these systems can protect the drug from acid-induced degradation in the stomach, modulate drug release and improve pharmacokinetic properties of the drug [6]. In particular, poly (lactic-co-glycolic acid) (PLGA), poly (D,L-lactide) (PDLLA), and poly ( $\varepsilon$-caprolactone) (PCL) have been widely used as drug carriers owing to their biocompatibility and biodegradability [7].

The utilization of different polymeric carriers to prepare LTZ formulations with improved characteristics has been reported. LTZ-PLGA particles have been prepared with drug entrapment efficiencies reaching up to $82 \%$ and a release profile that was greatly influenced by particle size distribution, with faster release and burst effects occurring when polydispersity of the particles is high $[8,9,10,11]$. Whilst the definition of 
'monodispersity' is not fully established, superior performance of particles is commonly attributed to their narrow size distribution; in this case and according to many reports, such particles are denoted as 'monodisperse'. It has been realized that, designing monodisperse particulate systems could be advantageous compared to their higher size distribution (polydisperse) counterparts, in terms of providing uniform drug loading, controlled drug release and consistent pharmacokinetic properties. Accordingly, monodisperse particles (MDP) can potentially maximize benefits of therapy and reduce adverse drug effects [12, $13,14,15,16,17,18]$. Furthermore, uniform distribution of the drug within monodisperse polymeric carriers may facilitate the prediction of drug release by calculating the overall surface area of the particulate system with respect to its volume, and the equivalent distance between the diffused drug particles and/or the rate of degradation of the carrier. Therefore, the progression towards in vivo studies would ascertain its effectiveness as that demonstrated by in vitro testing. The reliability on these calculations may decrease as the polydispersity of particles increases $[12,13]$.

Many previous studies have reported on the benefits of carrier systems possessing narrow particle size distribution. For example, in pulmonary drug delivery systems, designing MDP can maximize their delivery to the target region of the lung and reduce the undesirable deposition in the upper airways $[19,20]$. Likewise, systemic routes of administration such as in case of intravenous (IV) or intramuscular (IM) delivery of anticancer drugs in monodisperse systems can facilitate targeted embolization of tumor vessels [21]. 
Monodisperse drug delivery systems have been designed via chemical methods, e.g. by using a hypercross linking technique to prepare stable monodisperse particles [14]. Alternatively, physical approaches can also be employed. The Vibrating Orifice Aerosol Generator (VOAG) is an apparatus introduced by Berglund and Liu and is designed to produce a constant jet of monodisperse liquid droplets that dry off upon passing through a drying column, leaving the solutes that were dissolved in that liquid in the form of MDP [22]. To the best of our knowledge, this technology has not yet been explored to prepare solid MDP, owing to a number of limitations, such as low product yield, fluctuation of liquid pressure that builds upon passing through the tubes of the apparatus, clogging of the vibrating orifice, inefficient drying of the liquid feed, and difficulty of collecting the generated particles.

We have recently reported on the preparation and characterization of letrozole-loaded PDLLA particles for drug delivery in breast cancer therapy using the conventional emulsion-solvent evaporation method [23]. In the present work, we have adapted the modified VOAG technology, aiming to generate solid MDP using PCL or PDLLA polymeric carriers loaded with the anticancer drug, LTZ. Production parameters such as drug loading, organic phase concentration, liquid flow rate, organic: aqueous phase ratio, stirring rate and others were screened to explore their effects on particle size and particle size distribution utilizing Plackett-Burman experimental design. The product yield, microscopic morphology and thermal properties of the solid MDP were studied. Furthermore, following dispersion in aqueous medium, particle size, size distribution and drug loading were investigated, and drug release was evaluated over a period of one month, 
release data were fitted to a range of kinetic models to elucidate the underlying release mechanism. To evaluate the potential of the VOAG-generated LTZ-loaded MDP, long term cytotoxicity studies were conducted using the cancerous breast cell lines MCF-7 in comparison to the normal mammary epithelial cells (MEC). The findings of this study were appraised in light of the progress made with anticancer drug delivery using polymeric systems. To the best of our knowledge, this is the first study that utilized the VOAG system with the aim of designing monodisperse polymeric microparticles for providing prolonged drug release.

\section{Materials and methods}

\subsection{Materials}

Letrozole (LTZ; purity 99\%) was purchased from Jiangsu Sainty Handsome Co, Ltd, China. Poly (D, L-Lactic acid) (PDLLA; MW 15,000) and poly ( $\varepsilon$-caprolactone) (PCL; MW 50,000) were purchased from Polysciences, Inc, PA, USA. Dichloromethane (HPLC gradient grade, 99.8\%), polyvinyl alcohol (PVA; 88\% hydrolyzed), polystyrene monodisperse microparticles $(20 \mu \mathrm{m})$, and dialysis tubing cellulose membranes (MW cutoff $=14,000 \mathrm{Da}$ ) were all purchased from Sigma-Aldrich Co. LLC, Germany. Acetonitrile gradient grade was obtained from Merck Co, Germany, and Transcutol ${ }^{\circledR}$ HP was obtained from Gattefossé, Lyon, France. MCF-7 (ATCC® HTB-22 ${ }^{\mathrm{TM}}$ ) cell line, primary mammary epithelial cells (MEC), normal Eagle's Minimum Essential Medium (EMEM), mammary epithelial cell basal medium, mammary epithelial cell growth kit, penicillin-streptomycin- 
amphotericin B solution, trypsin-EDTA Solution, trypsin neutralizing solution, Dulbecco's Phosphate Buffered Saline (D-PBS) were all purchased from ATCC ${ }^{\circledR}$, VA, USA. All chemicals were used as received, without any further purification or chemical modification.

\subsection{Methods}

\subsubsection{Preparation of polymeric microparticles using VOAG technology}

As illustrated in Figure 1, a modified VOAG system was used to enhance its efficiency in generating solid MDP. This was achieved by stabilizing the liquid pressure; thus, the Teflon O-ring ( $0.075 \mathrm{~mm}$ in thickness) that holds the orifice disc was replaced with a rubber O-ring ( $2 \mathrm{~mm}$ in thickness). To overcome clogging of the orifice, the orifice disc having a hole diameter of $20 \mu \mathrm{m}$ was replaced with a specially manufactured orifice disc purchased from Lenox Laser, Inc. (MD, USA) with the following parameters: disc diameter: $9.5 \mathrm{~mm}$ $\pm 0.26 \% \mathrm{~mm}$, hole diameter: $300 \mu \mathrm{m} \pm 5 \% \mu \mathrm{m}$, thickness: $0.05 \mathrm{~mm} \pm 5 \% \mathrm{~mm}$, and centering: $\pm 0.25 \mathrm{~mm}$. To overcome the inefficient drying that may lead to difficulty in collecting solid particles, the drying column was removed and the liquid orifice cup was inverted and mounted on a beaker containing a dispersion medium of $0.04-1 \% \mathrm{w} / \mathrm{v}$ PVA aqueous solution.

A solution of 1 or $2 \% \mathrm{w} / \mathrm{v}$ of PCL or PDLLA in DCM was prepared to which an amount equivalent to 5 or $30 \% \mathrm{w} / \mathrm{w}$ of LTZ was dissolved. The resultant organic solution was loaded into a syringe connected to the pump of the VOAG apparatus adjusted at a flow rate of $0.17-1.7 \mathrm{~mL} / \mathrm{min}$. Upon passing through the $300 \mu \mathrm{m}$ orifice vibrating at a frequency of 
$100-1000 \mathrm{kHz}$, a constant stream of monodisperse droplets was generated. The droplets were collected in a $0.04-1 \% \mathrm{w} / \mathrm{v}$ PVA aqueous medium which served as a stabilizer to prevent particle aggregation [24]. The ratio of the organic to aqueous media was in the range of 1:4 -1:8 v/v. The resultant dispersion was stirred at 250-500 rpm, at ambient temperature for $24 \mathrm{~h}$ to ensure complete solvent evaporation. Solidified microparticles were harvested through filtration, washed several times with distilled water to remove residual PVA, centrifuged at $8,500 \mathrm{rpm}$ and $5^{\circ} \mathrm{C}$ for $10 \mathrm{~min}$, and finally dried under vacuum at $-19 \mathrm{kPa}$ at $35^{\circ} \mathrm{C}$ for $48 \mathrm{~h}$.

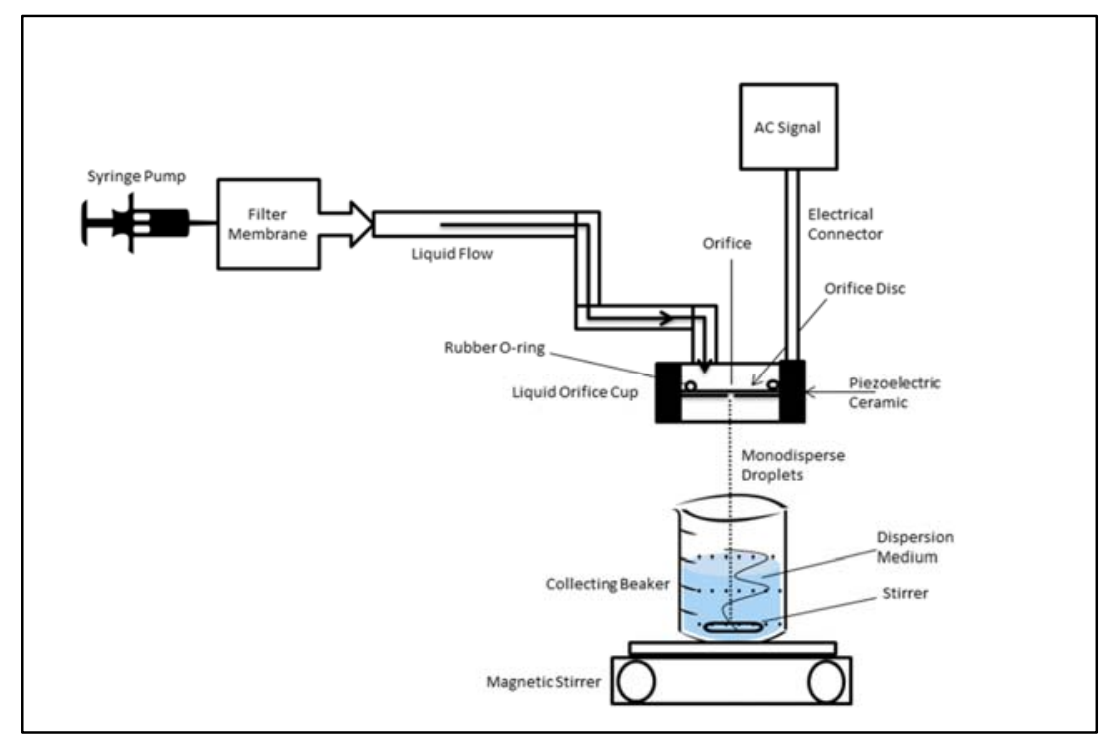

Figure 1. A schematic presentation of the modified VOAG made to manufacture PCL and PDLLA based LTZ-loaded monodisperse microparticles.

\subsubsection{Experimental Design for Optimizing the Production Parameters}

Using Minitab ${ }^{\circledR} 17$ Statistical Software (Coventry, UK), a 12-run, 2-level Plackett-Burman experimental design was created with each run containing a combination of two levels 
(high and low) of the following factors (independent variables): drug loading (X1), organic phase concentration (X2), organic: aqueous phase ratio (X3), PVA concentration (X4), frequency of the vibrating orifice (X5), liquid flow rate (X6), and stirring rate (X7) which are affecting particle size and specifically, particle size distribution (Y) (dependant variable). The highest and lowest levels for each independent variable were selected based on the reported results of previous studies in literature $[25,26,27]$. Table 1 lists the conditions of the 12 experiments with all of the different experimental. Each run was done in triplicate for each of the utilized polymeric carriers; PCL and PDLLA. Particles generated from each run were analyzed for their particle size distribution where the average span values were calculated and reported.

Table 1: Plackett-Burman Design for Optimizing PCL- and PDLLA-based Formulations

\begin{tabular}{lccccccc}
\hline $\begin{array}{c}\text { Formul } \\
\text {-ations }\end{array}$ & $\begin{array}{c}\mathbf{X 1} \\
(\% \mathbf{w} / \mathbf{w})\end{array}$ & $\begin{array}{c}\mathbf{X 2} \\
(\% \mathbf{w} / \mathbf{v})\end{array}$ & $\mathbf{X 3}$ & $\begin{array}{c}\mathbf{X 4} \\
(\mathbf{\%} / \mathbf{v})\end{array}$ & $\begin{array}{c}\mathbf{X 5} \\
(\mathbf{k H z})\end{array}$ & $\begin{array}{c}\mathbf{X 6} \\
(\mathbf{m l} / \mathbf{s e c})\end{array}$ & $\begin{array}{c}\mathbf{X 7} \\
(\mathbf{r p m})\end{array}$ \\
\hline F1 & 5 & 1 & $1: 8$ & 0.1 & 1000 & 0.17 & 500 \\
$\mathbf{F 2}$ & 30 & 2 & $1: 4$ & 0.1 & 100 & 0.17 & 250 \\
$\mathbf{F 3}$ & 30 & 1 & $1: 8$ & 0.04 & 100 & 0.17 & 500 \\
$\mathbf{F 4}$ & 5 & 2 & $1: 8$ & 0.04 & 1000 & 0.17 & 250 \\
F5 & 30 & 2 & $1: 4$ & 0.1 & 1000 & 0.17 & 500 \\
F6 & 5 & 2 & $1: 8$ & 0.1 & 100 & 1.7 & 500 \\
F7 & 30 & 2 & $1: 8$ & 0.04 & 1000 & 1.7 & 250 \\
F8 & 5 & 2 & $1: 4$ & 0.04 & 100 & 1.7 & 500 \\
F9 & 5 & 1 & $1: 4$ & 0.04 & 100 & 0.17 & 250 \\
F10 & 5 & 1 & $1: 4$ & 0.1 & 1000 & 1.7 & 250 \\
F11 & 30 & 1 & $1: 4$ & 0.04 & 1000 & 1.7 & 500 \\
F12 & 30 & 1 & $1: 8$ & 0.1 & 100 & 1.7 & 250 \\
\hline
\end{tabular}




\subsubsection{Scanning electron microscopy studies}

Scanning electron microscopy (SEM) studies were conducted by spreading 5-10 $\mathrm{mg}$ of the collected dried particles on a double-sided tape fixed on an aluminum holder, followed by spray-coating the sample with a gold film (thickness around $20 \mathrm{~nm}$ ). Imaging was carried out at $15.0 \mathrm{kV}$ using Nova NanoSEM ${ }^{\circledR} 450$ scanning electron microscope (FEI, California, USA).

\subsubsection{Differential scanning calorimetry}

Thermal characteristics of the VOAG-generated solid particles were investigated using Differential Scanning Calorimetry (DSC) (DSC 8000, Perkin Elmer Co., USA) equipped with an intra-cooling system (Intra-cooler II, Perkin Elmer Co., USA). Samples (2-4 mg) of each formulation were placed into the DSC aluminum pans, each tightly sealed with an aluminum cover. Heating scans were conducted over a temperature range of $0-200^{\circ} \mathrm{C}$ or $-70-200^{\circ} \mathrm{C}$ for PDLLA- and PCL-based formulations, respectively, at a heating rate of $10^{\circ} \mathrm{C} / \mathrm{min}$. Samples were cooled down to either $-70{ }^{\circ} \mathrm{C}$ or $0^{\circ} \mathrm{C}$ and then heated up to 200 ${ }^{\circ} \mathrm{C}$ to record the DSC thermograms. Nitrogen gas flow was in operation throughout the experiments at $40 \mathrm{~mL} / \mathrm{min}$ to eliminate humidity that might otherwise interfere with the thermal scanning. Pure samples of LTZ, PCL and PDLLA as well as LTZ-PCL, and LTZPDLLA formulations were analyzed, with the aim of evaluating the crystalline properties of the samples. 


\subsubsection{Crystalline structure investigations using X-ray diffraction (XRD)}

Crystalline structure was further evaluated using X-ray diffraction (D8 Advance diffractometer, Bruker Co, Germany) via employing $\mathrm{CuK} \alpha$ radiation source. Pure LTZ, PCL and PDLLA as well as LTZ-PCL, and LTZ-PDLLA formulations were analyzed using $\mathrm{A} 1^{\circ}$ divergence slit between the $2 \theta$ range $5-60^{\circ} \mathrm{C}$ with a step size of $0.1^{\circ} \mathrm{C}$ and step time of $1 \mathrm{sec}$. DIFFRAC.EVA software was used to present the XRD patterns.

\subsubsection{Particle size analysis using laser diffraction}

Powder samples were dispersed in deionized water and analyzed at a temperature of $25^{\circ} \mathrm{C}$ by using the Malvern Mastersizer 2000 laser diffraction instrument (Malvern Instruments Ltd, UK). Particle size and size distribution were determined by measuring the median volume diameter (MVD) and span, respectively. The mean values of three samples obtained from three different batches were calculated for each formulation. Span was calculated based on the formula: $\operatorname{Span}=(\mathrm{D} 90-\mathrm{D} 10) / \mathrm{D} 50$, where D90, D10, and D50 represent the median diameter by which $90 \%, 10 \%$, and $50 \%$ of the size distribution has a smaller particle size than the given value of the equation $[28,29,30]$.

\subsubsection{Determination of drug loading and entrapment efficiency}

Drug loading was determined by solubilizing specific quantities of the different formulations in $50 \mathrm{ml}$ acetonitrile, followed by sonication for $10 \mathrm{~min}$ to ensure complete dissolution of the drug, and then LTZ concentration was measured via Ultra Performance Liquid Chromatography (UPLC) (Waters Co., USA). In all experiments, the mobile phase 
used was acetonitrile and water $(35: 65 \mathrm{v} / \mathrm{v})$, flowing at a rate of $0.3 \mathrm{ml} / \mathrm{min}$, UV was set at $240 \mathrm{~nm}$, the column used was ACQUITY UPLC ${ }^{\circledR}$ BEH C18, $1.7 \mu \mathrm{m}$, and the column and sample temperature was set at $25^{\circ} \mathrm{C}$. Entrapment efficiency (EE) was determined by measuring the concentration of free drug (unentrapped) in the dispersion medium as reported previously [23]. In summary, LTZ-loaded microparticles dispersed in water was centrifuged using Sorvall ${ }^{\circledR}$ Stratos $^{\circledR}$ Centrifuge (Fisher Scientific, USA) at $8500 \mathrm{rpm}$ $(14,400 \mathrm{~g}),-5{ }^{\circ} \mathrm{C}$ for 60 min using Centrisart ${ }^{\circledR}$ I tube (Sartorius $\mathrm{GmbH}$, Germany), which consist of filter membrane (molecular weight cut-off 5,000 Da). The separated aqueous supernatant was then mixed with acetonitrile and assayed for unentrapped drug concentration by UPLC-UV set at $240 \mathrm{~nm}$. The column used was ACQUITY UPLC® BEH $\mathrm{C} 18,1.7 \mu \mathrm{m}$, and the temperature was set at $25^{\circ} \mathrm{C}$. The particles along with encapsulated LTZ remained in the outer chamber while the suspended and dissolved LTZ in the aqueous phase moved into the sample recovery chamber through the filter membrane. The amount of assayed unentrapped LTZ was subtracted from initial amount of LTZ to calculate drug entrapment efficiency of NPs. The percentage EE was calculated as follows: EE $\%=$ (Amount of drug recovered/Total amount of Drug used) *100. The concentration of LTZ in the separated LTZ-loaded particles from supernatant was also quantified after extraction with dichloromethane to ensure the accuracy of determining the entrapment efficiency using the supernatant subtraction from the initial concentration of LTZ. The experiment was performed in triplicate for each batch and average drug EE was calculated. 


\subsubsection{In vitro drug release and kinetic modeling studies}

Drug release study was performed by using dialysis tubing cellulose membranes (MW cutoff 14,000 Da). Known quantities of the dried LTZ-polymer loaded microparticles were suspended in $10 \mathrm{ml}$ of the release medium which was composed of $0.1 \mathrm{M}$ phosphate buffer saline (PBS, pH 7.4) with $20 \% \mathrm{v} / \mathrm{v}$ Transcutol ${ }^{\circledR}$ and filled in the dialysis tubes that were then sealed with their specified closures. The tubes were immersed in closed glass containers filled with $200 \mathrm{~mL}$ of the diffusion medium. These containers were placed in a shaking water bath (JULABO Labortechnik GmbH, Seelbach, Germany) adjusted at $37 \pm 1{ }^{\circ} \mathrm{C}$ and 100 rotations per min for one month. At specific intervals, $5 \mathrm{~mL}$ of the external medium was withdrawn, filtered, and injected into the UPLC to determine LTZ concentration. Similar volumes of fresh medium were used to replace the withdrawn samples and maintain the sink condition. Data were fitted into a range of kinetic models, which are zero-order, first order, Higuchi model, Hixson-Crowell model, and KorsemeyerPeppas semi-empirical model. For each model, both release rate constants $(\mathrm{k})$ and correlation coefficients $\left(\mathrm{R}^{2}\right)$ were determined by using the corresponding equations [31]. The model that provided $\mathrm{R}^{2}$ values that are closer to unity was considered the release order.

\subsubsection{Cytotoxicity studies and treatment of cells}

MEC and MCF-7 cells were cultured in mammary epithelial cell basal medium fortified with mammary epithelial cell growth kit and EMEM medium, respectively in $25 \mathrm{~cm}^{2}$ flasks at $37^{\circ} \mathrm{C}$ and $5 \% \mathrm{CO}_{2}$. After one week, the cells were split and transferred into $75 \mathrm{~cm}^{2}$ flasks. The medium was changed every 2-3 days until the cells became $90-95 \%$ confluent and 
were then harvested and used for cytotoxicity experiments. Cells were seeded in 96-well plates at a density of 10,000 cells/well and then incubated for $24 \mathrm{~h}$ at $37^{\circ} \mathrm{C}$ and $5 \% \mathrm{CO}_{2}$ to enable the cells to recover and attach. After $24 \mathrm{~h}$ of incubation, cells were treated with 100 $\mathrm{nM}, 1 \mu \mathrm{M}, 10 \mu \mathrm{M}$, and $100 \mu \mathrm{M}$ of LTZ from each formulation. Cells were treated with pure LTZ or polymeric LTZ-loaded MDP in EMEM medium containing acetonitrile and Transcutol $^{\circledR}(1: 5 \mathrm{v} / \mathrm{v})$ to ensure complete solubility of LTZ in the medium after being released from the polymeric shell, while preserving the polymeric carriers intact. The 1:5 $\mathrm{v} / \mathrm{v}$ ratio was selected based on preliminary experiments (not shown) performed for optimal solubility. This was an important step to achieve an appropriate cellular uptake of LTZ. Consequently, to rule out the effects of the solvents on cell viability, three control groups were made. This was an important step to achieve an appropriate cellular uptake of LTZ. Simultaneously, three control groups were used to examine their individual as well as collective effect on cell viability. The first group consisted of acetonitrile and EMEM medium $(1: 5 \mathrm{v} / \mathrm{v})$, the second consisted of Transcutol ${ }^{\circledR}$ and EMEM medium $(5: 1 \mathrm{v} / \mathrm{v})$, and the third consisted of EMEM medium with a mixture of acetonitrile and Transcutol ${ }^{(1: 5}$ $\mathrm{v} / \mathrm{v}$ ). Additionally, the effects of the polymeric carriers (PCL or PDLLA) were assessed by treating the MCF-7 cells with each blank polymer that contains no drug (the control group in Figures 8 and 9). Cytotoxicity was evaluated using nuclear staining after incubation of the treated cells for a period of $48 \mathrm{~h}$. 


\subsubsection{Cytotoxicity measurement based on nuclear staining}

Following completion of treatment, MEC or MCF-7 cells were fixed with $4 \% \mathrm{v} / \mathrm{v}$ formaldehyde, followed by $0.1 \% \mathrm{v} / \mathrm{v}$ 4',6-Diamidino-2-phenylindole dihydrochloride (DAPI) staining. Cell number was assessed through automated assessment of nuclear count using ArrayScan ${ }^{\mathrm{TM}}$ XTI Live High Content Platform (Thermo-Fisher Scientific, NY, USA) where 25 fields per well were selected for analysis. Number of viable cells was assessed by automated quantitation of DAPI positive nuclei using target activation module (HCS Studio® Cell Analysis Software), following exclusion of apoptotic nuclei distinguished by their collapsed size, higher chromatin intensities and nuclear fragmentation [32].

\subsubsection{Statistical analysis}

Results were reported as mean \pm Standard Deviation (SD) for the different parameters assessed. Student's $t$-tests and One-Way Analysis of Variance (ANOVA) were used to compare between the independent variables, for two groups and more than two sets of data, respectively. For this purpose, IBM Statistical Package for Social Sciences (IBM SPSS ${ }^{\circledR}$ Statistics - version 21) was used, and the difference was considered statistically significant when the calculated p-value was less than 0.05. Minitab ${ }^{\circledR} 17$ Statistical Software (Coventry, UK) was used to run the Plackett-Burman experimental design. 


\section{Results and discussion}

\subsection{Experimental Design for Optimizing the Production Parameters}

Multiple factors involved in the production process of MDP were reported to have an effect on particle size distribution including drug loading, organic phase concentration, organic: aqueous phase ratio, PVA concentration, frequency of the vibrating orifice, liquid flow rate, and stirring rate [33]. Therefore, it was essential to study the effects of these factors on particle size distribution in order to determine the optimal values of each one that would yield optimal MDP. Thus, a screening of those factors that were thought to have an impact on particle size distribution was undertaken based on Plackett-Burman design which is a powerful tool that allows the accurate identification of major factors influencing an outcome with the least number of experiments. This is because Plackett-Burman design is a fractional factorial design that combines the advantage of accuracy of full factorial designs and the few experimental runs of fractional factorial designs [25, 26, 27].

A 12-run, 2-level Plackett-Burman design was created with each run containing a different combination of high or low levels of each factor as listed previously in Table 1 . The different factor combinations resulted in a wide variation in both particle size and particle size distribution. As listed in Table 2, the average particle size ranged from 15.6 $\pm 1.1 \mu \mathrm{m}$ to $91.6 \pm 2.0 \mu \mathrm{m}$ for PCL-based formulations, whereas PDLLA-based formulations had a particle size range of $22.7 \pm 0.70 \mu \mathrm{m}$ to $99.6 \pm 3.1 \mu \mathrm{m}$. A large variation in particle size distribution was also observed among the different formulations in which the span values 
ranged from $0.22 \pm 0.02$ to $1.24 \pm 0.27$ and from $0.29 \pm 0.04$ to $1.48 \pm 0.36$ in PCL-based and PDLLA-based formulations, respectively (Table 2).

Table 2: Summary of the particle size and particle size distribution analyses for formulations run in Plackett-Burman design

\begin{tabular}{c|cc|cc}
\hline \multirow{2}{*}{ Formulation } & \multicolumn{2}{|c|}{ PCL-based Microparticles } & \multicolumn{2}{c}{ PDLLA-based Microparticles } \\
\cline { 2 - 5 } & $\begin{array}{c}\text { Mean diameter } \\
(\boldsymbol{\mu m})\end{array}$ & Span & $\begin{array}{c}\text { Mean diameter } \\
(\boldsymbol{\mu m})\end{array}$ & Span \\
\hline F1 & $47.9 \pm 1.9$ & $0.52 \pm 0.03$ & $59.2 \pm 1.1$ & $0.60 \pm 0.02$ \\
F2 & $47.0 \pm 2.4$ & $0.56 \pm 0.03$ & $53.3 \pm 3.2$ & $0.59 \pm 0.04$ \\
F3 & $33.4 \pm 2.7$ & $0.37 \pm 0.03$ & $44.0 \pm 3.4$ & $0.44 \pm 0.04$ \\
F4 & $58.7 \pm 1.5$ & $0.60 \pm 0.02$ & $64.0 \pm 2.3$ & $0.59 \pm 0.07$ \\
F5 & $15.6 \pm 1.1$ & $0.22 \pm 0.02$ & $22.7 \pm 0.70$ & $0.29 \pm 0.07$ \\
F6 & $91.6 \pm 2.0$ & $1.24 \pm 0.27$ & $99.6 \pm 3.1$ & $1.48 \pm 0.36$ \\
F7 & $47.1 \pm 2.4$ & $0.58 \pm 0.04$ & $58.0 \pm 5.6$ & $0.61 \pm 0.05$ \\
F8 & $57.9 \pm 1.8$ & $0.60 \pm 0.04$ & $61.1 \pm 4.8$ & $0.61 \pm 0.09$ \\
F9 & $61.8 \pm 3.2$ & $0.61 \pm 0.05$ & $46.6 \pm 2.3$ & $0.57 \pm 0.09$ \\
F10 & $76.7 \pm 2.4$ & $0.88 \pm 0.03$ & $84.2 \pm 2.9$ & $0.93 \pm 0.04$ \\
F11 & $17.4 \pm 1.2$ & $0.26 \pm 0.04$ & $24.0 \pm 2.6$ & $0.29 \pm 0.04$ \\
F12 & $88.7 \pm 1.3$ & $1.15 \pm 0.22$ & $97.8 \pm 3.0$ & $1.46 \pm 0.36$ \\
\hline
\end{tabular}

Regression analysis allowed the accurate elucidation of the effects of each factor on particle size distribution; span (Y) through the following equation for PCL-based formulations: $\mathrm{Y}_{\text {span-PCL }}=0.397-0.00876 \mathrm{X} 1+0.0033 \mathrm{X} 2+0.0561 \mathrm{X} 3+4.296 \mathrm{X} 4-0.000273 \mathrm{X} 5+$ 0.1983 X6 - 0.000773 X7. On the other hand, PDLLA-based formulations had a different equation, but with a similar pattern to these observed in PCL-based formulations. The equation illustrating the effects of each factor on particle size distribution; span (Y) for PDLLA-based formulations was: $Y_{\text {span-PDLLA }}=0.169-0.00724 \mathrm{X} 1+0.0211 \mathrm{X} 2+0.0786$ 
$\mathrm{X} 3+6.26 \mathrm{X} 4-0.000338 \mathrm{X} 5+0.2505 \mathrm{X} 6-0.000702 \mathrm{X} 7$. Both equations were highly accurate in predicting the span values for each formulation through setting the parameters/factors at their optimal levels to ensure achieving the lowest span values possible before running the experiment. As shown in Figure 2, a strong correlation was established between the predicted and observed span values using these equations with a multiple regression coefficient $\left(\mathrm{R}^{2}\right)$ equivalent to 0.9668 and 0.9521 for PCL- and PDLLAbased formulations, respectively.
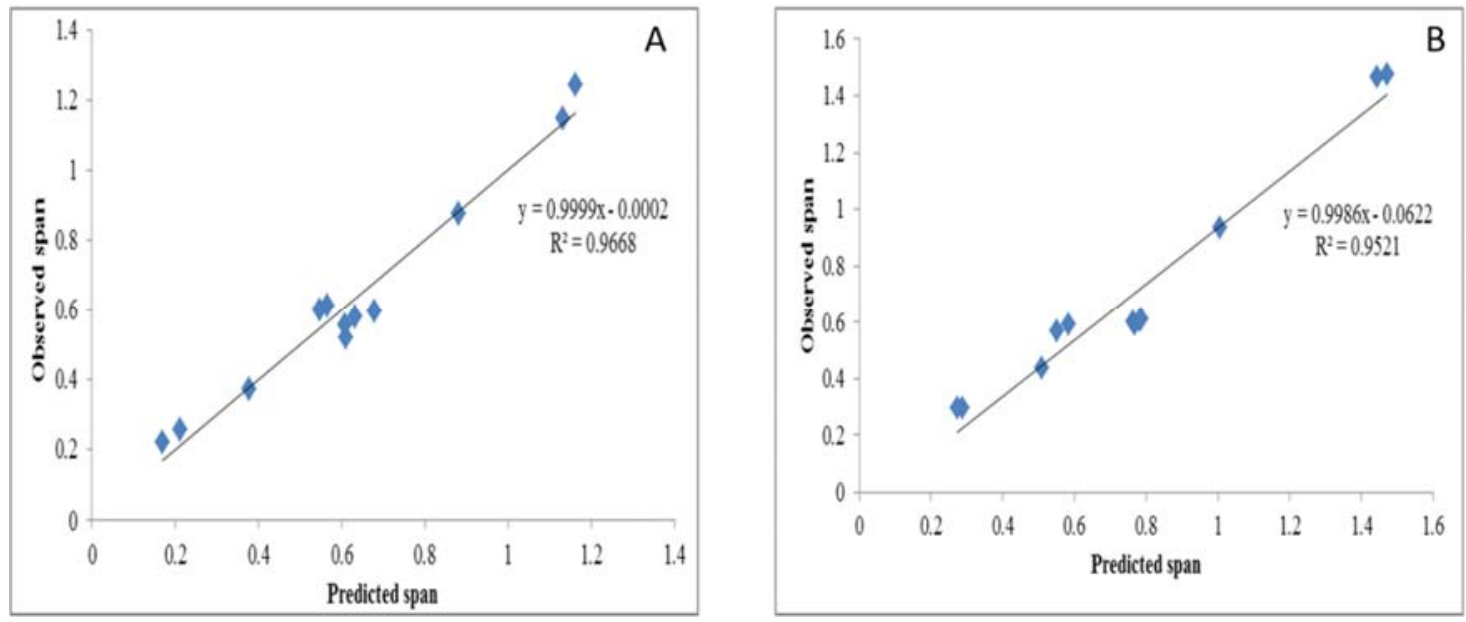

Figure 2: Correlation between observed and predicted span values by the model for (A): PCL- based microparticles and (B): PDLLA-based microparticles.

ANOVA statistical analyses revealed that all production parameters/factors had a significant effect on particle size distribution $(p<0.05)$, except for organic phase concentration X2 $(p=0.955)$ in PCL-based formulations (Table 3). However, drug loading (X1), organic phase concentration (X2), and stirring rate $(\mathrm{X} 7)$ were found to have no significant impact on particle size distribution when PDLLA was used (Table 3). Such 
interesting observations were made previously when different polymeric carriers were used owing to the differences in their intrinsic characteristics $[25,26,27,33,34,35,36]$.

Table 3: Results of one-way analysis of variance of the effects of the different production factors on particle size distribution

\begin{tabular}{lc|c|c|c}
\hline \multirow{2}{*}{ Factor } & \multicolumn{2}{c|}{ PCL-based formulations } & \multicolumn{2}{c}{ PDLLA-based formulations } \\
\cline { 2 - 5 } & Coefficient & $\boldsymbol{p}$-value & Coefficient & $\boldsymbol{p}$-value \\
\hline Drug loading (\%w/w) - X1 & -0.1094 & 0.017 & -0.0906 & 0.086 \\
Organic phase conc. (\%w/v) - X2 & 0.0017 & 0.955 & 0.0106 & 0.804 \\
Organic/aqueous phase - X3 & 0.1122 & 0.015 & 0.1572 & 0.017 \\
PVA concentration (\%w/v) - X4 & 0.1289 & 0.009 & 0.1878 & 0.009 \\
Frequency (kHz) - X5 & -0.1228 & 0.011 & -0.1522 & 0.019 \\
Liquid flow rate (m1/sec) - X6 & 0.1517 & 0.005 & 0.1917 & 0.009 \\
Stirring rate (rpm) - X7 & -0.0967 & 0.025 & -0.0878 & 0.093 \\
\hline
\end{tabular}

The mean of the triplicates has been used to compute the coefficient and $\mathrm{p}$-values for each factor.

Coefficient values indicated that some of the factors had a positive relationship with the span, meaning that the increase in these parameters led to the increase in the span. These included: organic phase concentration (X2), organic: aqueous phase ratio (X3), PVA concentration (X4), and liquid flow rate (X6). In contrast, the other parameters had an inverse relationship with the span, meaning an increase in these parameters caused a reduction in the span, which was favorable. These included drug loading $(\mathrm{X} 1)$, frequency of the vibrating orifice (X5), and stirring rate (X7). Such findings were also in agreement with other previous reports $[25,33]$.

There was a general agreement between PCL- and PDLLA-based formulations in terms of the order of the production parameters affecting their particle size distribution. As shown in Figure 3, for PCL-based formulations, the order of the parameters from those with 
highest impact to those with lowest impact was: Liquid flow rate $(\mathrm{X} 6)>$ PVA concentration $(X 4)>$ Frequency of the vibrating orifice $(X 5)>$ Organic: aqueous phase ratio $(X 3)>$ Drug loading $(\mathrm{X} 1)>$ Stirring rate $(\mathrm{X} 7)>$ Organic phase concentration $(\mathrm{X} 2)$. On the other hand, with PDLLA-based formulations, the order of the parameters was: Liquid flow rate (X6) $>$ PVA concentration $(\mathrm{X} 4)>$ Organic: aqueous phase ratio $(\mathrm{X} 3)>$ Frequency of the vibrating orifice $(\mathrm{X} 5)>$ Drug loading $(\mathrm{X} 1)>$ Stirring rate $(\mathrm{X} 7)>$ Organic phase concentration (X2). This indicated that there was a minor difference between the two polymers in terms of the parameters affecting their particle size distribution. However, it would be more appropriate to consider each polymeric carrier independently during the production process in order to achieve optimal results.
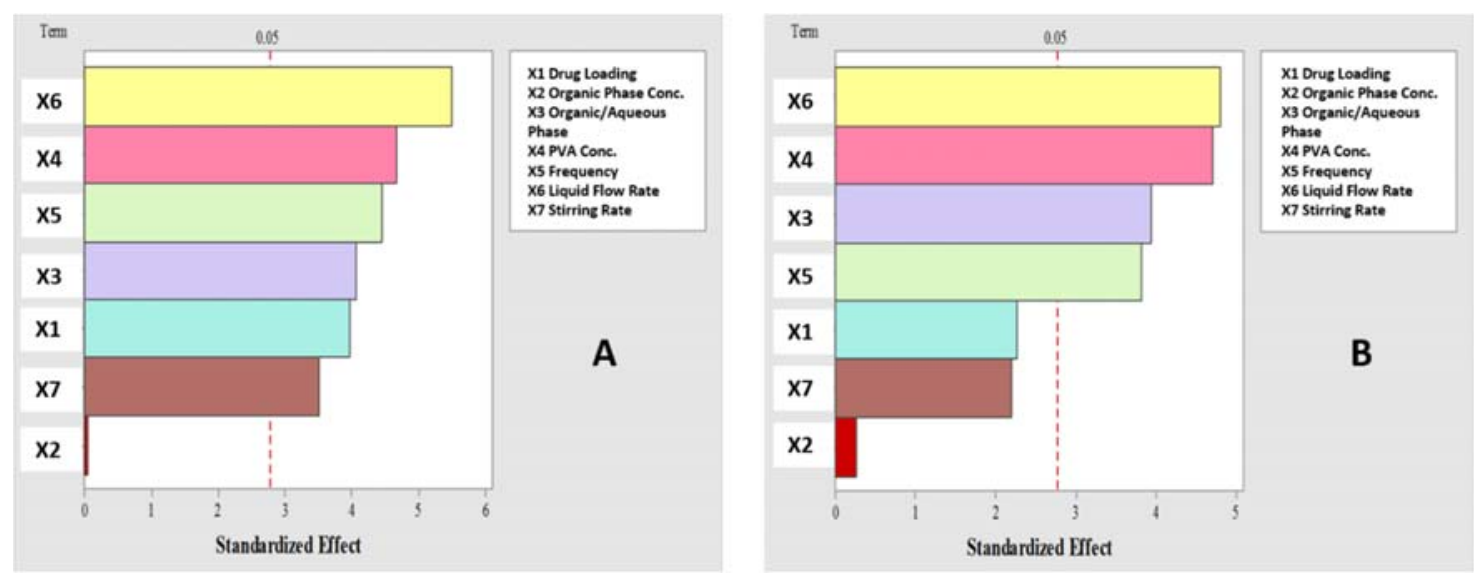

Figure 3. Pareto chart for the different production factors affecting particle size distribution as expressed in span. (A): PCL-based formulations, (B): PDLLA-based formulations. The higher the length of the bars, the more effect the factor has on span. Bars crossing $p$-value $=$ 0.05 indicate statistical significance.

Generally, the increase in drug loading caused a decrease in the span due to the subsequent increase in the viscosity of the dispersing particles. This was attributed to the fact that the 
content of LTZ increased in relation to the polymer (PCL or PDLLA) with higher drug loadings, causing a net increase in the overall viscosity of the formulation. As such, during partitioning to the external aqueous phase, more viscous organic droplets tend to resist the applied stirring forces as compared to the opposed dilute organic droplets, making them maintain their shape, size, and homogeneity [37, 38, 39]. In contrast, the increase in organic: aqueous phase ratio led to an increased span. This was because the larger volume of aqueous external phase relative to the organic phase induced higher potential of agglomeration between the partitioning particles, resulting in unalike droplets, and consequently polydisperse particles [33].

The use of PVA was crucial in producing well dispersed droplets during the emulsification process. However, the concentration of PVA (X4) should be determined carefully since concentrations lower than those required produced agglomerated particles, whereas higher concentrations, increased the external resistive forces against the droplets being emulsified [40]. The lowest span achieved in this study, was obtained with PVA concentrations of $0.04 \% \mathrm{w} / \mathrm{v}$, and the highest span was obtained with PVA concentrations of $0.1 \% \mathrm{w} / \mathrm{v}$.

The frequency of the vibrating orifice (X5) significantly impacted the particle size distribution. Higher frequency tends to be more effective in generating monodisperse microparticles. This was mainly due to the intensive forces applied on the emerging droplets from the VOAG, which produced smaller particles that were easier to disperse homogenously than larger counterparts [41]. Similarly, liquid flow rate (X6), determining how much the VOAG received from the organic phase to generate the microparticles had 
a significant effect on the span. This was anticipated since larger volumes supplied per unit time would produce larger particles that were less likely to uniformly disperse within the aqueous phase, leaving the span at its highest values (165).

Finally, higher stirring rates were more efficient in maintaining an adequately stable dispersion of the droplets, leading to narrower particle size distribution [42]. Table 4 lists the optimal production parameters identified based on statistical analyses of the PlackettBurman design. In some experiments, however, different drug loading percentages were used in order to examine their effects on certain outcomes as would be discussed in the upcoming sections.

Table 4: Optimal values of the different production factors for yielding the optimal particle size distribution as suggested by the model generated from Plackett-Burman design.

\begin{tabular}{cccccccc}
\hline Factor & $\begin{array}{c}\text { Drug } \\
\text { loading } \\
(\% \mathrm{w} / \mathrm{w}) \\
\mathrm{X} 1\end{array}$ & $\begin{array}{c}\text { Organic } \\
\text { phase conc. } \\
(\% \mathrm{w} / \mathrm{v}) \\
\mathrm{X} 2\end{array}$ & $\begin{array}{c}\text { Organic/ } \\
\text { aqueous } \\
\text { phase } \\
\mathrm{X} 3\end{array}$ & $\begin{array}{c}\text { PVA } \\
\text { conc. } \\
(\% \mathrm{w} / \mathrm{v})\end{array}$ & $\begin{array}{c}\text { Frequency } \\
(\mathrm{kHz})\end{array}$ & $\begin{array}{c}\text { Liquid } \\
\text { flow rate } \\
(\mathrm{ml} / \mathrm{sec})\end{array}$ & $\begin{array}{c}\text { Stirring } \\
\text { rate } \\
(\mathrm{rpm})\end{array}$ \\
$\begin{array}{c}\text { Optimum } \\
\text { value }\end{array}$ & 30 & 1 & $1: 4$ & 0.04 & 1000 & 0.17 & 500 \\
\hline
\end{tabular}

\subsection{Morphological evaluation of VOAG-generated particles}

One important limitation in the development of biodegradable polymer microparticles for controlled-release drug delivery applications has been the difficulty of specifically designing systems exhibiting precisely controlled release rates. Because microparticle size is a primary determinant of drug release, we developed a methodology for controlling release kinetics employing MDP. As shown in Figure 4, the SEM showed that powder 
particles using either PCL or PDLLA carriers were spherical in shape with apparently fine smooth surfaces and in the micrometer size range, regardless of LTZ concentration in the formulation. All particles appeared uniform in size and dimensions, indicating that the VOAG technology can generate monodisperse microparticles when PCL or PDLLA were used as polymeric carriers and irrespective of LTZ amount loaded. However, particles with $5 \%$ LTZ loading were smaller than those with higher drug loading $(30 \%)$, which can be ascribed to the increased viscosity of the feed solution when the higher LTZ concentration was used, possibly resulting in larger droplets accommodating larger amount of dissolved drug and polymer [43]. Nevertheless, regardless of formulation composition, particles did not form agglomerates, indicating that the amount of PVA used was appropriate for providing homogeneous dispersion of the polymeric particles. Other investigators have successfully developed monodisperse chitosan-enriched particles for gene therapy using an approach referred to as the double walled microsphere technology [42, 44].

\subsection{Particle size analysis}

Subsequent to size observation with SEM, laser diffraction studies were performed to provide a more robust analysis of size and size distribution of the polymeric microparticles. As listed in Table 5, the median particle size, also referred to as the volume median diameter (VMD), was found to be dependent on LTZ concentration $(p<0.5)$. Thus, the measured size increased from $10.9 \pm 0.21 \mu \mathrm{m}$ to $24.7 \pm 0.82 \mu \mathrm{m}$ when LTZ loading was increased from $0 \%$ to $30 \%(\mathrm{w} / \mathrm{w})$ in PCL-based formulations. 

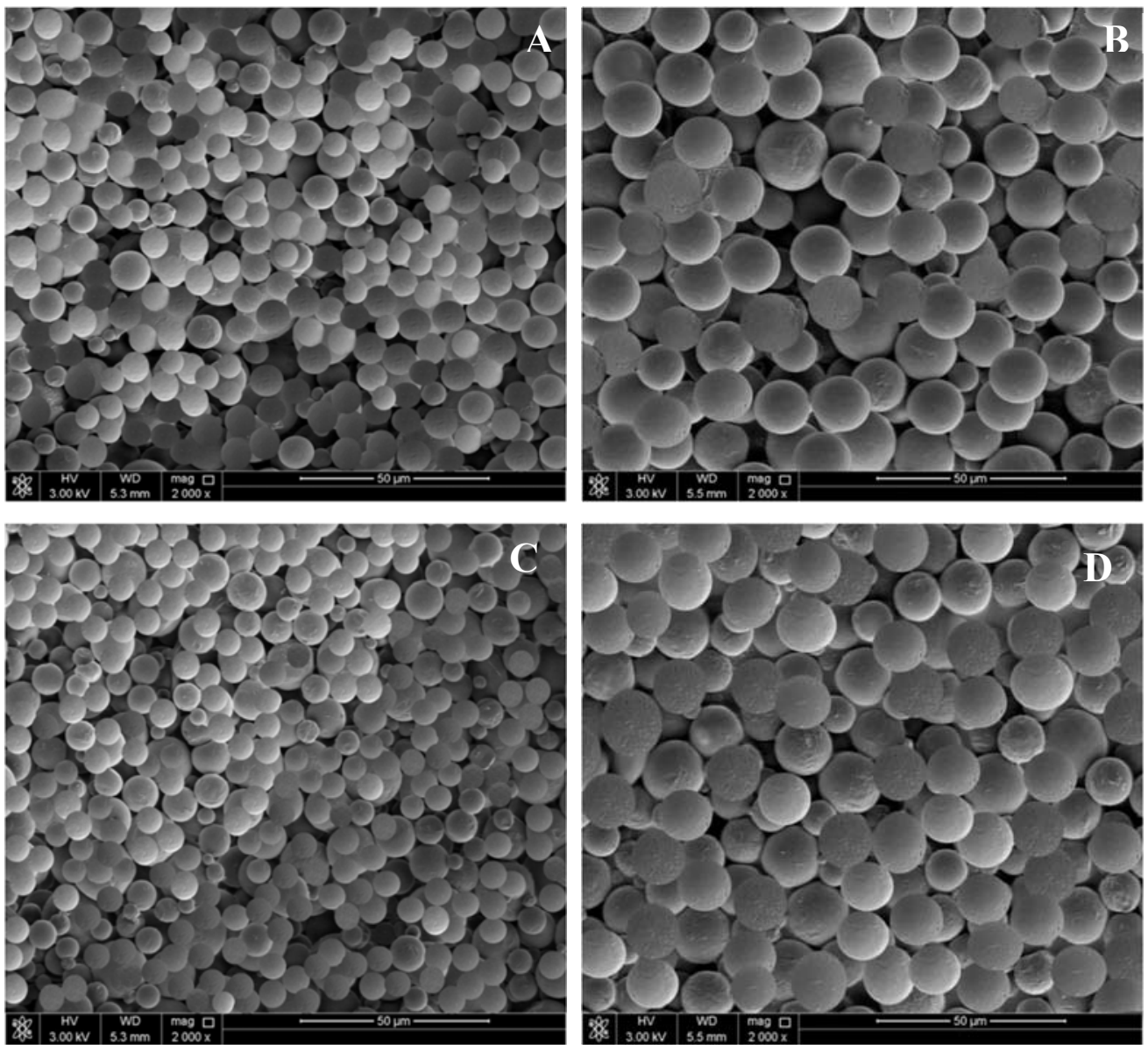

Figure 4. SEM images of PCL and PDLA based formulations of LTZ. (A): PCL 5\% LTZ; (B): PCL 30\% LTZ; (C): PDLLA 5\% LTZ; (D): PDLLA 30\% LTZ. Each image is typical for the observation taken from three different batches.

Likewise, there was an increase in the measured size from $12.9 \pm 0.49 \mu \mathrm{m}$ to $27.3 \pm 0.87$ $\mu \mathrm{m}$ when LTZ loading was increased from $0 \%$ to $30 \% \mathrm{w} / \mathrm{w}$ in PDLLA-based formulations. Size findings, measured by laser diffraction, agree with SEM observations. The significant increase in particle size $(p<0.05)$ might be attributed to the increase in viscosity of the VOAG feed solution due to the higher LTZ concentration, resulting in higher resistance of the solution to fragmentation into small aerosol droplets [45]. There was no significant 
difference between particle size of PCL and PDLLA formulations that were loaded with $5 \%(\mathrm{w} / \mathrm{w}) \mathrm{LTZ}$, whereas those particles prepared using the PDLLA polymer with $30 \%$ $(\mathrm{w} / \mathrm{w}) \mathrm{LTZ}$ were significantly larger than PCL formulation counterpart.

Size distribution in our VOAG technology studies were comparable to those measured for standard polystyrene monodisperse microparticles. Upon analysis, the VMD of the polystyrene particles were found to have a size of $23.4 \pm 2.50 \mu \mathrm{m}$ and their span was 0.167 \pm 0.02 , suggesting better monodispersity (i.e. narrower size distribution) of our polymeric particles manufactured using the VOAG technology. The advantage of monodisperse particles over polydisperse counterparts has been established in terms of insuring a predictable controlled drug release [46]. Thus, our investigations established the applicability of the VOAG technology for generating highly MDP when PCL or PDLLA are used as carriers and LTZ is used as a model drug.

Table 5. Particle size (VMD) and particle size distribution (Span) for optimized polymeric formulations. Span $=(90 \%$ undersize $-10 \%$ undersize $) /$ VMD.

\begin{tabular}{lcc}
\hline Formulation & VMD $(\boldsymbol{\mu m})$ & Span \\
\hline PCL & $10.9 \pm 0.21^{*}$ & $0.171 \pm 0.002$ \\
PCL 5\% (w/w) LTZ & $14.7 \pm 0.67^{*}$ & $0.185 \pm 0.01$ \\
PCL 30\% (w/w) LTZ & $24.7 \pm 0.82^{*}$ & $0.162 \pm 0.01$ \\
PDLLA & $12.9 \pm 0.49^{*}$ & $0.179 \pm 0.002$ \\
PDLLA 5\% (w/w) LTZ & $16.3 \pm 1.1^{*}$ & $0.195 \pm 0.01$ \\
PDLLA 30\% (w/w) LTZ & $27.3 \pm 0.87^{*}$ & $0.178 \pm 0.01$ \\
Polystyrene Control & $23.4 \pm 2.50$ & $0.167 \pm 0.02$ \\
\hline
\end{tabular}

Data presented as mean $\pm \mathrm{SD}, \mathrm{n}=3$.

$(*)$ : Indicates statistical significance at $p=0.05$ between same polymer-based groups. 


\subsection{Drug loading and entrapment efficiency studies}

Drug loading approached almost $100 \%$ (98.1 to $99.3 \%)$ in all formulations, indicating negligible drug losses during production, which highlights the economic feasibility of the VOAG technology and the successful adaptation of the apparatus. Importantly, the entrapment efficiency, representing the actual amount of drug incorporated within the polymeric carriers, was also high and significantly increased by increasing LTZ loading from $5 \%(\mathrm{w} / \mathrm{w})$ to $30 \%(\mathrm{w} / \mathrm{w})$. For each drug concentration, the entrapment of LTZ was higher $(p<0.05)$ in PCL formulations compared to those of PDLLA; nevertheless, the entrapment values were considerably high regardless of drug concentration and polymer type. Thus, the drug entrapment was $89.9 \% \pm 0.08$ and $94.1 \% \pm 0.32$ for PDLLA-based formulations containing LTZ concentrations of $5 \% \mathrm{w} / \mathrm{w}$ and $30 \% \mathrm{w} / \mathrm{w}$, respectively. Similarly, the entrapment increased from $92.4 \% \pm 0.25$ to $96.8 \% \pm 0.06$ for PCL-based formulations when LTZ concentrations was increased from $5 \% \mathrm{w} / \mathrm{w}$ to $30 \% \mathrm{w} / \mathrm{w}$. The direct relationship between drug concentration and entrapment efficiency could be attributed to the increase in particle size with increased drug concentration (Figure 4; Table 5). It is hypothesized here that larger size offers smaller surface area exposed to the outer aqueous phase, hence greater drug proportions can successfully be accommodated by the particles and initial drug leakage (i.e. burst effect) is retarded. Thus, diffusion of the drug from the polymeric system to the aqueous medium would be much lower in large particles compared to smaller particle, for each polymer. A previous study with LTZ incorporated into polymeric formulations prepared using emulsion-solvent evaporation have shown that 
larger particles may have lower affinity to the aqueous medium, preserving more drug within the polymeric matrix than smaller particles [47].

\subsection{Physical structure investigations using DSC and XRD}

As shown in Figure 5, DSC thermograms of pure LTZ revealed its crystalline nature with a melting point of $186^{\circ} \mathrm{C}$, while PDLLA was confirmed to be amorphous with no melting point peak. On the other hand, PCL was confirmed to be crystalline with a melting point of $63.5^{\circ} \mathrm{C}$. The incorporation of LTZ into PCL or PDLLA resulted in conversion of the drug into its amorphous form since the melting peak disappeared from all formulations' thermograms. XRD studies were conducted to back up the DSC investigations. As shown in Figure 6, XRD diffractograms showed few distinctive peaks of LTZ crystals in formulations containing $20 \% \mathrm{w} / \mathrm{w}, 25 \% \mathrm{w} / \mathrm{w}$, and $30 \% \mathrm{w} / \mathrm{w} \mathrm{LTZ}$, which can be attributed to drug crystals lying on top of particle surfaces. Although drug entrapment efficiency was higher in these formulations as compared to the ones having only $5-10 \% \mathrm{w} / \mathrm{w} \mathrm{LTZ}$, the proposed drug crystals on the surface of particles might indicate that the drug concentration exceeded its solubility in the polymeric matrix, leaving some particles in their crystalline form near or on the top of the surface $(48,49)$. Thus, findings in this study combined with previous observations indicate that incorporation of crystalline hydrophobic drugs into hydrophobic matrices is likely to convert the drug into its amorphous counterpart in a manner that depends on carrier properties and drug physicochemical characteristics and drug concentration in the formulation. 

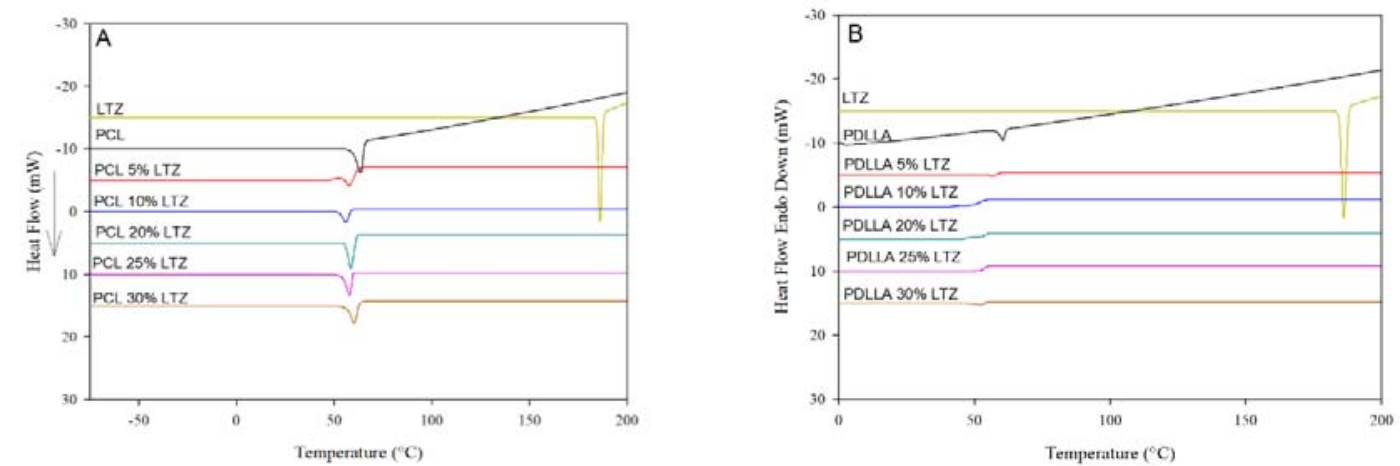

Figure 5. DSC thermograms of (A): pure LTZ, pure PCL, and LTZ-PCL formulations, and (B): pure LTZ, pure PDLLA, and LTZ-PDLLA formulations. The profiles presented are typical for three independent measurements using three different batches.
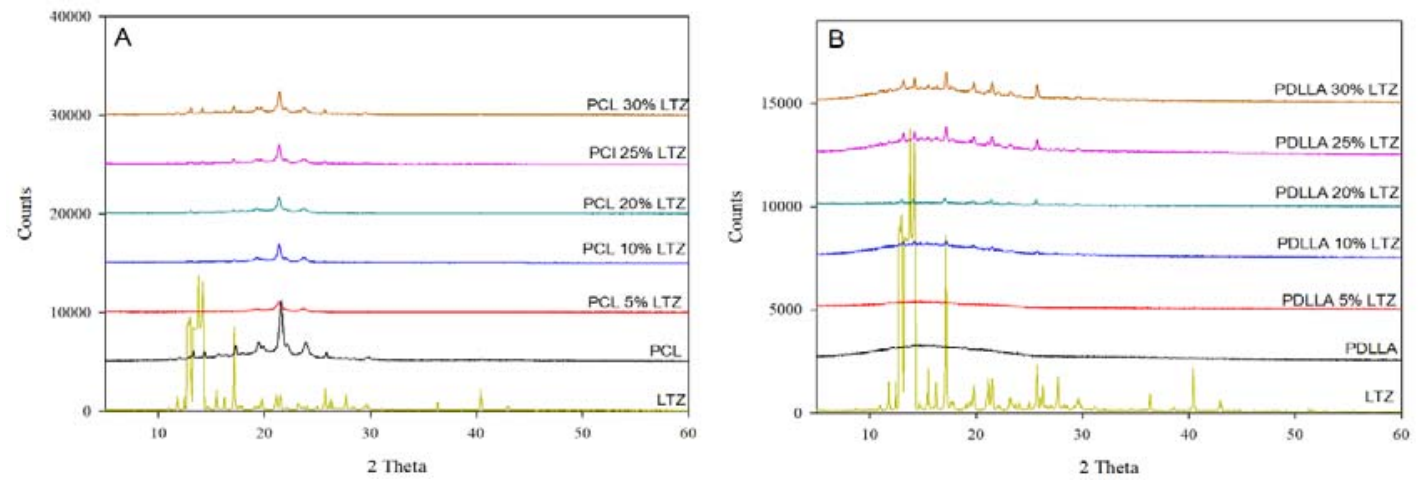

Figure 6. X-Ray diffractograms of (A) pure LTZ, pure PCL, and LTZ-PCL formulations, and (B) pure LTZ, pure PDLLA, and LTZ-PDLLA formulations. The profiles presented are typical for three independent measurements using three different batches.

\subsection{In vitro drug release and kinetic modeling investigations}

In vitro drug release study was conducted to investigate the ability of the monodisperse VOAG-generated MDP to provide controlled release of LTZ. In vitro release studies aim to predict the release of drug in vivo and its biodistribution profile [48]. The selected four 
formulations (PCL 5\% LTZ, PCL 30\% LTZ, PDLLA 5\% LTZ, and PDLLA 30\% LTZ) were investigated for drug release using dialysis membranes in $0.1 \mathrm{M}$ phosphate buffer solution ( $\mathrm{pH}$ 7.4) containing $20 \% \mathrm{v} / \mathrm{v}$ Transcutol $^{\circledR}$. Transcutol is an established cosurfactant/cosolvent used in drug release studies and permeation enhancement investigations $[49,50,51]$; thus, Transcutol ${ }^{\circledR}$ was used in the present study to facilitate the release of the hydrophobic drug LTZ from the polymeric matrix of the monodisperse PCL and PDLLA microparticles.

For the release study to be more robust and thorough, sampling was performed over a prolonged period of one month. The prolonged release study is believed to provide an advantage since previous investigations on polymeric formulations were limited to shorter periods of time. Thus, our LTZ formulations have been studied for potential long-term drug release (e.g. monthly administration) to constitute a base for further in vivo studies as possible intramuscular depot formulations, which are planned to be one of the dimensions of our future investigations.

Figure 7 represents the cumulative percentage of drug released over time for the four tested formulations. The release of LTZ was sustained in which up to $52.7 \%, 93 \%, 35.2 \%$, and $85.4 \%$ of the drug was released after 30 days from PCL 5\% LTZ, PCL 30\% LTZ, PDLLA 5\% LTZ, and PDLLA 30\% LTZ, respectively. The overall release of LTZ increased in correspondence with the increase in its loading and entrapment efficiency where particles with $30 \%$ LTZ content showed the highest release rates, whereas those with $5 \%$ LTZ 
content exhibited lower release rates as evident by the release rate constant $(\mathrm{k})$ values reported in Table 6.

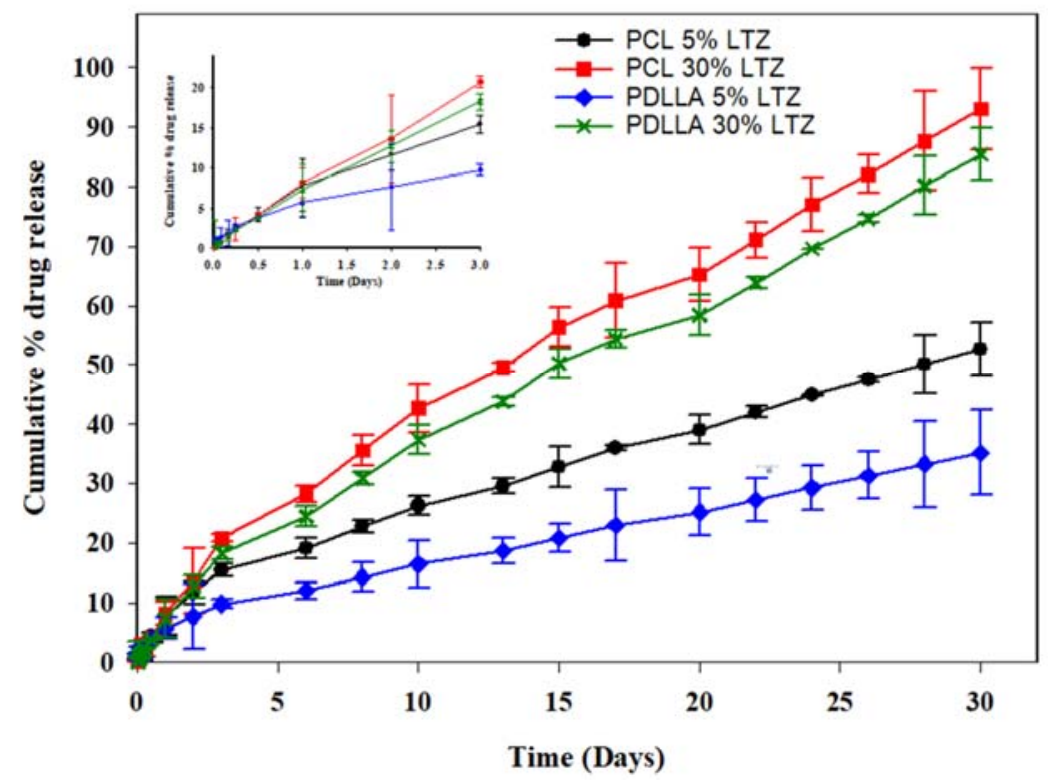

Figure 7. In vitro release profiles of $5 \%$ or $30 \% \mathrm{w} / \mathrm{w}$ LTZ loading in PCL and PDLLA microparticles. $\mathrm{n}=3$, bars represent $\mathrm{SD}$.

Table 6. Drug release kinetic modeling of formulations prepared using PCL or PDLLA polymers with $5 \%$ or $30 \%$ LTZ loading.

\begin{tabular}{|c|c|c|c|c|c|c|c|c|c|c|c|c|c|}
\hline \multirow[t]{2}{*}{ Formulation } & \multicolumn{2}{|c|}{$\begin{array}{c}\text { Zero order } \\
\text { (Phase 1) }\end{array}$} & \multicolumn{2}{|c|}{$\begin{array}{c}\text { Zero order } \\
\text { (Phase 2) }\end{array}$} & \multicolumn{2}{|c|}{ First order } & \multicolumn{2}{|c|}{ Higuchi } & \multicolumn{2}{|c|}{ Hixon-Crowell } & \multicolumn{3}{|c|}{ Korsemeyer-Peppas } \\
\hline & $\mathbf{K}$ & $\mathbf{R}^{2}$ & $\mathbf{K}$ & $\mathbf{R}^{2}$ & $\mathbf{K}$ & $\mathbf{R}^{2}$ & $\mathbf{K}$ & $\overline{\mathbf{R}^{2}}$ & $\mathbf{K}$ & $\mathbf{R}^{2}$ & $\mathbf{K}$ & $\mathbf{R}^{2}$ & $\mathbf{n}$ \\
\hline PCL 5\% LTZ & 5927.4 & 0.9951 & 1025.7 & 0.9939 & 0.040 & 0.9696 & 2.953 & 0.9833 & 0.002 & 0.3457 & 0.576 & 0.8203 & 0.11 \\
\hline PCL $30 \%$ LTZ & 5104.6 & 0.996 & 1803.7 & 0.9955 & 0.115 & 0.8531 & 5.224 & 0.9694 & -0.005 & 0.4623 & 0.563 & 0.8521 & 0.13 \\
\hline PDLLA 5\% LTZ & 4551.8 & 0.9867 & 708.1 & 0.9960 & 0.023 & 0.9695 & 1.876 & 0.9802 & -0.002 & 0.5967 & 1.230 & 0.9246 & 0.08 \\
\hline PDLLA $30 \%$ LTZ & 4533.7 & 0.9958 & 1751.1 & 0.9938 & 0.089 & 0.8878 & 4.736 & 0.9679 & -0.005 & 0.5533 & 0.549 & 0.8599 & 0.13 \\
\hline
\end{tabular}

The mean of the 6 replicates has been used to compute the $\mathrm{K}$ and $\mathrm{R}^{2}$ values for each model

The increase in drug entrapment efficiency within the polymeric matrix has been reported

to have an influence on drug release rate [52]. The higher the drug entrapment in polymeric 
particles, the greater is the propensity of the drug molecules to be present near the surfaces or close to the solid/liquid interface rather than the core of the polymeric particle. This hypothesis may constitute a justification for the increased rate of drug release from formulations that had higher drug loading [53]. The concentration of the drug might have exceeded its solubility in the polymer, resulting in coexistence of dissolved and undissolved forms of the drug, with the undissolved crystals protruding towards the surfaces of the particle, leading to faster drug release for the formulations loaded with higher LTZ concentrations. By contrast, in particles with lower drug loading, higher drug proportions are expected to be soluble or molecularly dispersed within the polymeric matrix, leading to predomination of the amorphous state of the drug, resulting in lower drug diffusion rates; this explanation is supported by the earlier XRD data presented in Figure 6. For each drug concentration, the release rate was higher in PCL-based microparticles compared to PDLLA-based preparations. This might be attributed to the smaller particles prepared upon using the PCL polymer, resulting in higher surface area of its particles and faster drug release from its matrix.

The extended release of LTZ for as long as one month suggested the potential of these formulations for intramuscular (IM) depot administration; however, this can be confirmed only if further in vivo experiments in the future supports this assumption. Data obtained from the in vitro release study were fitted to five empirical kinetic models: Zero-order, First order, Higuchi model, Hixson-Crowell model, and Korsemeyer-Peppas semiempirical model. Release rate constants $(\mathrm{k})$ and correlation coefficients $\left(\mathrm{R}^{2}\right)$ of the data and the corresponding kinetic models were computed for the four formulations and presented 
in Table 6. As shown in Figure 7, the release profiles of the four formulations were best fitted with a biphasic zero-order model where highest correlation coefficient values were obtained. The first phase lasted from 0-3 days, whilst the second started after 3 days and continued till the end of the study period (i.e. one month). Each phase had distinct release rate constants and correlation coefficients. It was observed that during the first phase, there was a slight burst effect ranging from $6.0 \%$ to $7.9 \%$, due to the rapid release of the drug molecules located at the solid/liquid interface of the polymeric particle. However, after 3 days, the burst effect decreased to a maximum of $2.4 \%$ since almost all drug molecules at the solid/liquid interface were depleted and the remaining drug portion within the particle cores started to be released in a more controlled manner. In other words, the path that the drug had to travel to reach the solid liquid interface is becoming longer. A depletion zone is created and expands as more drug is released

Burst effects were observed with PCL-based formulations compared to PDLLA-based particles, due to the relatively smaller particle size and higher surface area of the PCLbased microparticles. The burst release observed with our MDP was much lower than that seen in previous investigations where the proportions of drug lost due to burst effect in those studies were extremely high (e.g. exceeding $80 \%$ of the drug originally loaded). The higher burst release has been ascribed to the polydispersity of particles prepared in those studies and the fact that these were nanoparticles with higher surface area [11, 54]. Thus, our microparticles generated using the VOAG technology represent and advancement with a considerable advantage since the potential adverse effects that may occur due to the overwhelming burst effect would be diminished. The production of formulations with 
biphasic zero-order release kinetics entailed that as time elapsed, constant amounts of LTZ were released [55]. This is of high importance since these formulations were successful in modifying the release of LTZ to become linear, highly predictable, and consistent, which is one of the prime achievements of the present study.

\subsection{In Vitro Cytotoxicity Studies}

Cytotoxicity studies were conducted to evaluate the ability of our LTZ loaded MDP produced using the VOAG technology to inhibit the proliferation of MCF-7 human breast cancer cells in comparison to primary mammary epithelial cells (MEC). The studies were conducted by measuring the average number of viable cells after 24 and $48 \mathrm{~h}$ treatment with pure LTZ powder or VOAG-generated LTZ-loaded microparticles at a range of concentrations $(100 \mathrm{nM}, 1 \mu \mathrm{M}, 10 \mu \mathrm{M}$, and $100 \mu \mathrm{M})$. Both untreated as well as vehicletreated MCF-7 cells were used as controls to quantify the percentage of inhibition of cell proliferation.

As shown in Figure 8, results revealed that LTZ induced a concentration-dependent inhibition of cell proliferation, resulting in $11.3 \%, 39.2,48.8 \%$, and $74.6 \%$ decrease in cell number compared to untreated control $(p<0.05)$ at concentrations of $100 \mathrm{nM}, 1 \mu \mathrm{M}, 10$ $\mu \mathrm{M}$, and $100 \mu \mathrm{M}$, respectively. Similar findings have been reported previously [56]. During the first 24 hours of cells incubation and as expected, all concentrations of free LTZ showed higher cytotoxicity than drug-loaded MDP. After $48 \mathrm{~h}$ of incubation, the cells of free LTZ treated group began to re-proliferate and the attachment of cells became well at 72 hrs (data not shown), while the cell viability of LTZ-Loaded MDP group continued to 
decrease during the experimental period $(\mathrm{p}<0.05)$. This may suggest that the LTZ-loaded MDP showed sustained cell inhibition abilities against the MCF-7 cell lines. This phenomenon appears to correspond reasonably well to the in vitro drug controlled release properties. From the in vitro drug release experiment, it is clearly shown that LTZ can gradually be released from the microparticles in a sustained fashion for 30 days, while the free LTZ reached the concentration plateau in 12 to $24 \mathrm{~h}$. The sustained cytotoxicity of drug-loaded microparticles could be additionally attributed to the internalization of LTZloaded MDP into the cells and the successive drug release from microparticles inside the cells, enhancing the action of LTZ and preventing the short acute action of the drug. Similar findings regarding the uptake of Nor- $\beta$-lapachone- and Doxorubicin- PLGA loaded microparticles by treated cancer cells were previously reported $[57,58,59]$. Our results also indicated that our formulations were non-toxic to MEC normal breast epithelial cells at all concentrations expect for $100 \mu \mathrm{M}$, which indicates that these monodisperse polymeric formulations manufactured using the VOAG technology are potentially safe to noncancerous cells (Figure 9).

To the best of our knowledge, this study represents the first report employing the VOAG as a manufacturing tool of MDP. Bearing in mind that vibrating orifice technology has now reached the realm of manufacturing of novel drug delivery systems (presented in this report); the present investigation involving the VOAG technology is expected to open a gate for other studies involving other drugs and carrier systems. 


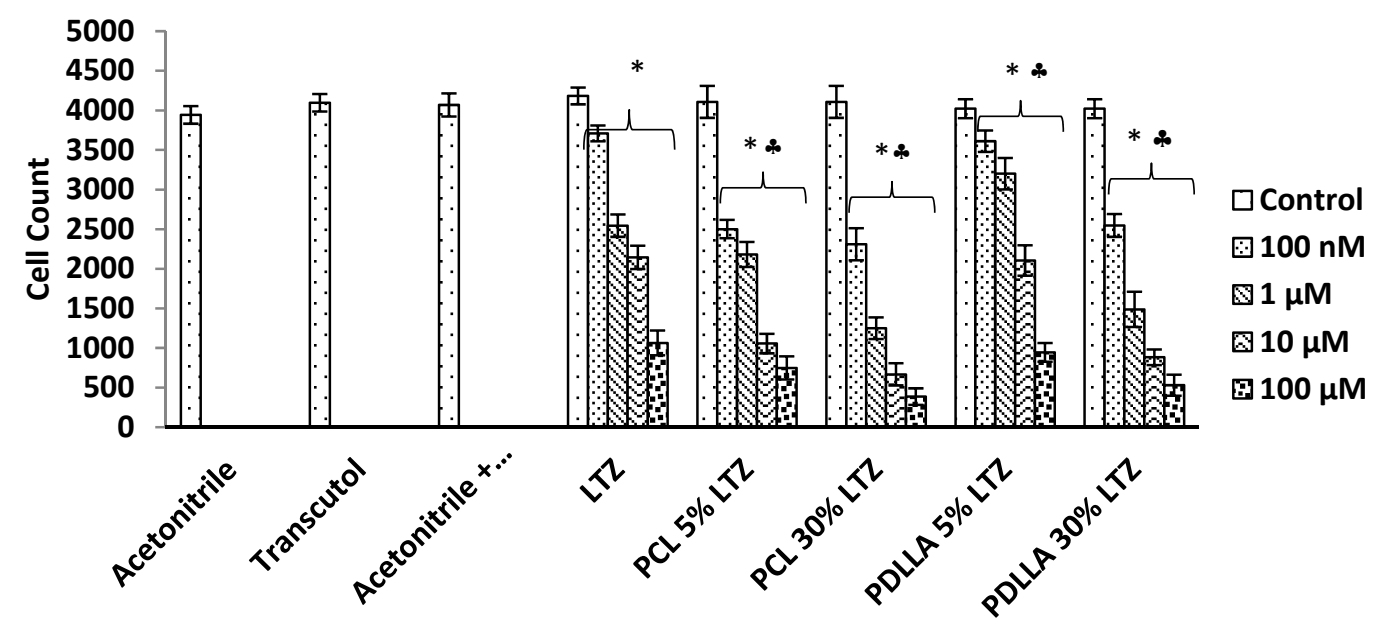

Figure 8. MCF-7 cell viability following $48 \mathrm{~h}$ treatment using different concentrations of LTZ microparticles prepared from PCL or PDLLA polymers. Bars represent the average number of nuclei of viable cells as compared with untreated controls. Cell number was assessed by automated quantitation of DAPI positive nuclei using ArrayScan XTI (Target activation module). Data presented as mean $\pm \mathrm{SD}, \mathrm{n}=3$. (*): Indicates statistical significance at $p<0.05$ compared to untreated cells (48-hrs post seeding). (*): Indicates statistical significance at $p<0.05$ compared to untreated cells ( $24 \mathrm{~h}$ post seeding)

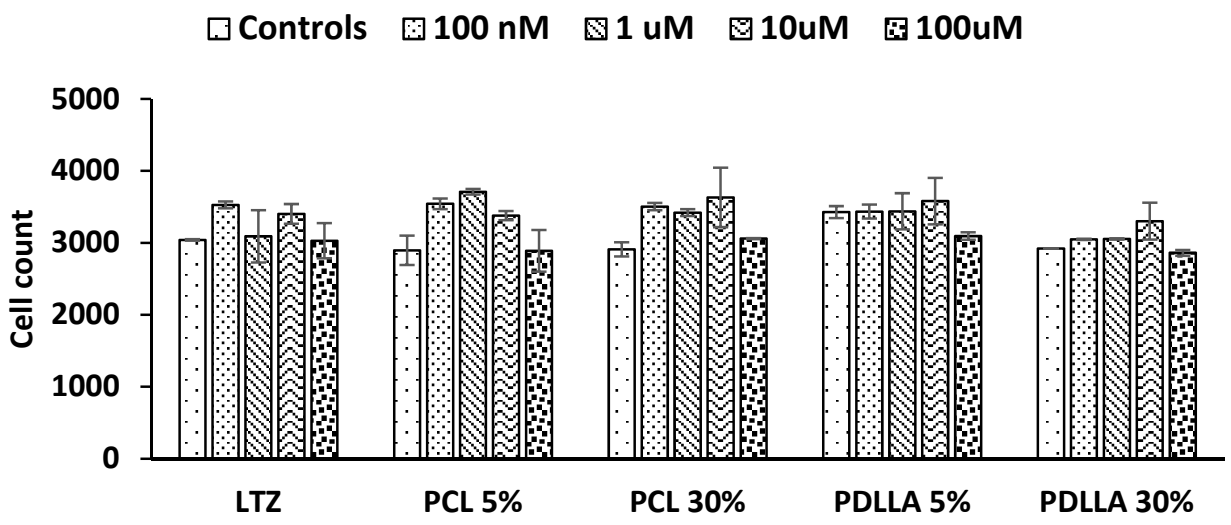

Figure 9. MEC cell viability following $48 \mathrm{~h}$ treatment using different concentrations of LTZ microparticles prepared from PCL or PDLLA polymers. Bars represent the average number of nuclei of viable cells as compared with untreated controls. Cell number was assessed by automated quantitation of DAPI positive nuclei using ArrayScan XTI (Target activation module). Data presented as mean $\pm \mathrm{SD}(\mathrm{n}=3)$. 


\section{Conclusion}

This study presented a novel approach of adopting a vibrating orifice technology for designing monodisperse polymeric microparticles. LTZ was incorporated within either PCL or PDLLA carriers in order to optimize its non-linear release profile in vitro. PlackettBurman experimental design was successful in identifying the most significant factors affecting particle size distribution to optimize the prepared particle. The resultant microparticles were characterized for particle size, size distribution, and morphology, which confirmed their narrow size distribution (Span values were smaller than those measured for monodispersed latex particles used for quality control and instrument calibration). LTZ loading approached $100 \%$, and entrapment efficiency values were very high for all formulations, reaching up to $94.1 \%$ and $96.8 \%$ for PDLLA and PCL formulations, respectively. Importantly, in vitro release studies revealed that LTZ release from the formulations was constant, time-independent, and followed bi-phasic zero-order kinetics. In vitro cytotoxicity showed that PCL and PDLLA formulations prolonged the cytotoxic effects of LTZ on MCF-7 cells at various concentrations. Importantly, these formulations showed no signs of toxicity on normal breast cells at all concentrations except for concentrations as high as $100 \mu \mathrm{M}$. The findings of this study are promising as they indicated that such MDP might serve as new LTZ formulations for monthly administration that offered better release profile, which may potentially result in minimal adverse effects. Future studies will include in vivo investigations using breast cancer animal models to explore the validity of our therapeutic assumption. 


\section{Acknowledgment}

This project was made possible by NPRP grant \# NPRP 4 - 496 - 3 - 157 from Qatar National Research Foundation (a member of Qatar Foundation) through its National Priorities Research Program granted to Dr. H. Younes. The statement(s) made herein are solely the responsibility of the authors. The authors would like to thank Dr. Saeed Al-Meer, Dr. Mohamed Yousef, and Mr. Essam Mohamed at the Central Lab Unit of Qatar University for their assistance with the SEM studies.

\section{Authors Contributions}

Bayan Alemrayat is the MSc student who performed the research experiments, analysis and collected data. Mohamed Elrayess guided and helped Bayan in conducting the in vitro cytotoxicity studies. Abdelbary Elhissi \& Raid Alany \& helped in supervising Bayan and commented on the manuscript writing. Husam Younes designed the research question, designed performed experiments, analysed data, supervised the progress of the project and wrote the final manuscript.

\section{References}

1. Simpson D, Curran MP, Perry CM. Letrozole: a review of its use in postmenopausal women with breast cancer. Drugs. 2004;64(11):1213-30.

2. Regan MM, Neven P, Giobbie-Hurder A, et al. Assessment of letrozole and tamoxifen alone and in sequence for postmenopausal women with steroid hormone receptor-positive breast cancer: the BIG 1-98 randomised clinical trial at $8 \cdot 1$ years median follow-up. The Lancet Oncology. 2011;12(12):1101-1108. doi: 10.1016/S1470-2045(11)70270-4.

3. The BIGCG. Letrozole Therapy Alone or in Sequence with Tamoxifen in Women with Breast Cancer. The New England journal of medicine. 2009;361(8):766-776. doi: 10.1056/NEJMoa0810818. PubMed PMID: PMC2921823.

4. Jin S-J, Jung JA, Cho S-H, et al. The pharmacokinetics of letrozole - its association with key body mass metrics. Int Journal of Clinical Pharmacology and Therapeutics. 2012;50(08):557565 . 
5. Cohen MH, Johnson JR, Li N, et al. Approval Summary : Letrozole in the Treatment of Postmenopausal Women with Advanced Breast Cancer. Clinical Cancer Research. 2002;8(3):665-669.

6. Grigoletto A, Maso K, Mero A, et al. Drug and protein delivery by polymer conjugation. Journal of Drug Delivery Science and Technology. 2016;32:132-141.

7. Mora-Huertas CE, Fessi H, Elaissari A. Polymer-based nanocapsules for drug delivery. International Journal of Pharmaceutics. 2010;385(1):113-142.

8. Hossain S, Islam S, Saha M, et al. Effect of Formulation Variables on the Release of Letrozole from Natural Biodegradable Polymeric Implants. British Journal of Pharmaceutical Research. 2014;4(20):2417-2435.

9. Mondal N, Pal TK, Ghosal SK. Development, physical characterization, micromeritics and in vitro release kinetics of letrozole loaded biodegradable nanoparticles. Pharmazie. 2008;63(5):361-365. doi: 10.1691/ph.2008.7365.

10. Mondal N, Pal TK, Ghosal SK. Development and validation of RP-HPLC method to determine letrozole in different pharmaceutical formulations and its application to studies of drug release from nanoparticles. Acta poloniae pharmaceutica. 2009;66(1):11-7.

11. Nerella A, Basava R, Devi A. Formulation, optimisation and in vitro characterisation of letrozole loaded solid lipid nanoparticles. International Journal of Pharmaceutical Sciences and Drug Research. 2014;6(3):183-188.

12. Cho SH, Park SY, Kim C, et al. Stabilization of monodispersed spherical silica particles and their alignment with reduced crack density. Colloids and Surfaces A: Physicochemical and Engineering Aspects. 2014;441:354-359.

13. Naeem M, Choi M, Cao J, et al. Effect of Particle Size on the Dissolution of Glibenclamide. American Heart Association Journal. 2015;26(1):869-879. doi: 10.1299/kikai1938.38.202.

14. Ouyang Y, Shi H, Fu R, et al. Highly monodisperse microporous polymeric and carbonaceous nanospheres with multifunctional properties. Aerosol Science and Technology. 2013;3(2):1430-1430.

15. Soares JS, Zunino P. A mixture model for water uptake, degradation, erosion and drug release from polydisperse polymeric networks. Biomaterials. 2010;31(11):3032-3042.

16. Wu HT, Ding CC, Chen KJ. Preparation of monodispersed PMMA particles and composite particles containing pigment green 36 by dispersion polymerization. Journal of the Taiwan Institute of Chemical Engineers. 2013;44(4):691-699. doi: 10.1016/j.jtice.2012.12.026.

17. Wu J, Kong T, Yeung KWK, et al. Fabrication and characterization of monodisperse PLGAalginate core-shell microspheres with monodisperse size and homogeneous shells for controlled drug release. Acta Biomaterialia. 2013;9(7):7410-7419. doi: 10.1016/j.actbio.2013.03.022.

18. Xia Y, Ribeiro PF, Pack DW. Controlled protein release from monodisperse biodegradable double-wall microspheres of controllable shell thickness. Journal of Controlled Release. 2013;172(3):707-714. doi: 10.1016/j.jconrel.2013.08.009.

19. Li H, Yu Y, Faraji Dana S, et al. Novel engineered systems for oral, mucosal and transdermal drug delivery. Journal of drug targeting. 2013;21(7):611-29.

20. Pawar VK, Singh Y, Meher JG, et al. Engineered nanocrystal technology: In-vivo fate, targeting and applications in drug delivery. Journal of Controlled Release. 2014;183:51-66. doi: 10.1016/j.jconre1.2014.03.030.

21. Yang $T$, Wan Z, Liu Z, et al. In situ mineralization of anticancer drug into calcium carbonate monodisperse nanospheres and their $\mathrm{pH}$-responsive release property. Materials Science and Engineering: C. 2016;63:384-392. doi: 10.1016/j.msec.2016.03.009. 
22. Berglund RN, Liu BYH. Generation of monodisperse aerosol standards. Environmental Science \& Technology. 1973;7(2):147-153.

23. Alemrayat B, Elhissi A, Younes HM. Preparation and characterization of letrozole-loaded poly(d,l-lactide) nanoparticles for drug delivery in breast cancer therapy. Pharmaceutical Development and Technology. 2018:1-8. doi: 10.1080/10837450.2018.1455698.

24. Quintanar-Guerrero D, Tamayo-Esquivel D, Ganem-Quintanar A, et al. Adaptation and optimization of the emulsification-diffusion technique to prepare lipidic nanospheres. European Journal of Pharmaceutical Sciences. 2005;26(2):211-218. doi: 10.1016/j.ejps.2005.06.001.

25. Awotwe-Otoo D, Zidan AS, Rahman Z, et al. Evaluation of anticancer drug-loaded nanoparticle characteristics by nondestructive methodologies. AAPS PharmSciTech. 2012;13(2):611-22. doi: 10.1208/s12249-012-9782-7.

26. Chauhan K, Trivedi U, Patel KC. Statistical screening of medium components by PlackettBurman design for lactic acid production by Lactobacillus sp. KCP01 using date juice. Bioresource Technology. 2007;98(1):98-103. doi: 10.1016/j.biortech.2005.11.017.

27. Vatanara A, Rouholamini Najafabadi A, Gilani K, et al. A Plackett-Burman design for screening of the operation variables in the formation of salbutamol sulphate particles by supercritical antisolvent. Journal of Supercritical Fluids. 2007;40(1):111-116. doi: 10.1016/j.supflu.2006.03.028.

28. Bindhu MR, Umadevi M. Synthesis of monodispersed silver nanoparticles using Hibiscus cannabinus leaf extract and its antimicrobial activity. Spectrochimica Acta - Part A: Molecular and Biomolecular Spectroscopy. 2013;101:184-190. doi: 10.1016/j.saa.2012.09.031.

29. Kamat V, Marathe I, Ghormade V, et al. Synthesis of Monodisperse Chitosan Nanoparticles and in Situ Drug Loading Using Active Microreactor. ACS Applied Materials \& Interfaces. 2015:151008120710000-151008120710000. doi: 10.1021/acsami.5b05100.

30. Selomulya C, Liu W, Wu WD, et al. Uniform chitosan microparticles prepared by a novel spray-drying technique. International Journal of Chemical Engineering. 2011;2011. doi: $10.1155 / 2011 / 267218$.

31. Ramteke KH, Dighe PA, Kharat AR, et al. Mathematical Models of Drug Dissolution : A Review. Scholars Academic Journal of Pharmacy. 2014;3(5):388-396.

32. Cummings BS, Wills LP, Schnellmann RG. Measurement of Cell Death in Mammalian Cells. Current protocols in pharmacology / editorial board, SJ Enna (editor-in-chief) [et al]. 2004;0 12:10.1002/0471141755.ph1208s25. doi: 10.1002/0471141755.ph1208s25. PubMed PMID: PMC3874588.

33. Mainardes RM, Evangelista RC. PLGA nanoparticles containing praziquantel: Effect of formulation variables on size distribution. International Journal of Pharmaceutics. 2005;290(1-2):137-144. doi: 10.1016/j.ijpharm.2004.11.027.

34. European Society for Medical Oncology | ESMO.

35. Kouchakzadeh H, Shojaosadati SA, Shokri F. Efficient loading and entrapment of tamoxifen in human serum albumin based nanoparticulate delivery system by a modified desolvation technique. Chemical Engineering Research and Design. 2014;92(9):1681-1692. doi: 10.1016/j.cherd.2013.11.024.

36. Rahman Z, Zidan AS, Habib MJ, et al. Understanding the quality of protein loaded PLGA nanoparticles variability by Plackett-Burman design. International Journal of Pharmaceutics. 2010;389(1-2):186-194. doi: 10.1016/j.ijpharm.2009.12.040.

37. Bi X, Hemar Y, Balaban MO, et al. The effect of ultrasound on particle size, color, viscosity and polyphenol oxidase activity of diluted avocado puree. Ultrasonics Sonochemistry. 2015;27:567-575. doi: 10.1016/j.ultsonch.2015.04.011. 
38. Shewan HM, Stokes JR. Analytically predicting the viscosity of hard sphere suspensions from the particle size distribution. Journal of Non-Newtonian Fluid Mechanics. 2015;222:72-81. doi: 10.1016/j.jnnfm.2014.09.002.

39. Wang X, Su MX, Cai XS. Effects of Material Viscosity on Particle Sizing by Ultrasonic Attenuation Spectroscopy. Procedia Engineering. 2015;102:256-264. doi: 10.1016/j.proeng.2015.01.141.

40. Rama Rao GV, López GP, Bravo J, et al. Monodisperse mesoporous silica microspheres formed by evaporation-induced self assembly of surfactant templates in aerosols. Advanced Materials. 2002;14(18):1301-1304. doi: 10.1002/1521-4095(20020916)14:18<1301::AIDADMA1301>3.0.CO;2-T.

41. Berkland C, Kim K, Pack DW. Fabrication of PLG microspheres with precisely controlled and monodisperse size distributions. Journal of controlled release : official journal of the Controlled Release Society. 2001;73(1):59-74.

42. Xu Q, Hashimoto M, Dang TT, et al. Preparation of monodisperse biodegradable polymer microparticles using a microfluidic flow-focusing device for controlled drug delivery. Small. 2009;5(13):1575-1581. doi: 10.1002/smll.200801855.

43. Fava LW, Serpa PBS, Külkamp-Guerreiro IC, et al. Evaluation of viscosity and particle size distribution of fresh, chilled and frozen milk of Lacaune ewes. Small Ruminant Research. 2013;113(1):247-250. doi: 10.1016/j.smallrumres.2013.03.010.

44. Xu Q, Xia Y, Wang $\mathrm{CH}$, et al. Monodisperse double-walled microspheres loaded with chitosan-p53 nanoparticles and doxorubicin for combined gene therapy and chemotherapy. Journal of Controlled Release. 2012;163(2):130-135. doi: 10.1016/j.jconrel.2012.08.032.

45. Astete CE, Kumar CSSR, Sabliov CM. Size control of poly(d,l-lactide-co-glycolide) and poly(d,l-lactide-co-glycolide)-magnetite nanoparticles synthesized by emulsion evaporation technique. Colloids and Surfaces A: Physicochemical and Engineering Aspects. 2007;299(1):209-216. doi: 10.1016/j.colsurfa.2006.11.055.

46. Overbeek JTG. Monodisperse colloidal systems, fascinating and useful. Advances in Colloid and Interface Science. 1982;15(3-4):251-277. doi: 10.1016/0001-8686(82)80003-1.

47. Mondal N, Halder KK, Kamila MM, et al. Preparation, characterization, and biodistribution of letrozole loaded PLGA nanoparticles in Ehrlich Ascites tumor bearing mice. International Journal of Pharmaceutics. 2010;397(1-2):194-200. doi: 10.1016/j.ijpharm.2010.06.049.

48. Balzus B, Colombo M, Sahle FF, et al. Comparison of different in vitro release methods used to investigate nanocarriers intended for dermal application. International Journal of Pharmaceutics. 2016;513(1):247-254. doi: 10.1016/j.ijpharm.2016.09.033.

49. Godwin DA, Kim N-H, Felton LA. Influence of Transcutol ${ }^{\circledR}$ CG on the skin accumulation and transdermal permeation of ultraviolet absorbers. European Journal of Pharmaceutics and Biopharmaceutics. 2002 2002/01/01/;53(1):23-27. doi: https://doi.org/10.1016/S09396411(01)00215-6.

50. Manconi M, Caddeo C, Sinico C, et al. Ex vivo skin delivery of diclofenac by transcutol containing liposomes and suggested mechanism of vesicle-skin interaction. European Journal of Pharmaceutics and Biopharmaceutics. 2011 2011/05/01/;78(1):27-35. doi: https://doi.org/10.1016/j.ejpb.2010.12.010.

51. Wu L, Qiao Y, Wang L, et al. A Self-microemulsifying Drug Delivery System (SMEDDS) for a Novel Medicative Compound Against Depression: a Preparation and Bioavailability Study in Rats. AAPS PharmSciTech. 2015;16(5):1051-1058. doi: 10.1208/s12249-014-0280-y. PubMed PMID: PMC4674636. 
52. Mandal B, Dey SK, Bhowmik M, et al. Development and in vitro evaluation of Letrozole loaded biodegradable nanoparticles for breast cancer therapy. Brazilian Journal of Pharmaceutical Sciences. 2009;45:585-591.

53. Budhian A, Siegel SJ, Winey KI. Controlling the in vitro release profiles for a system of haloperidol-loaded PLGA nanoparticles. International Journal of Pharmaceutics. 2008;346(12):151-159.

54. Kumar Jana S. Letrozole and Curcumin Loaded-PLGA Nanoparticles: A Therapeutic Strategy for Endometriosis. Journal of Nanomedicine \& Biotherapeutic Discovery. 2014;04(01):1-10.

55. Dekyndt B, Verin J, Neut C, et al. How to easily provide zero order release of freely soluble drugs from coated pellets. International Journal of Pharmaceutics. 2015;478(1):31-38. doi: 10.1016/j.ijpharm.2014.10.071.

56. Azria D, Larbouret $\mathrm{C}$, Cunat $\mathrm{S}$, et al. Letrozole sensitizes breast cancer cells to ionizing radiation. Breast Cancer Research. 2005;7(1):R156-R163.

57. Çağlar GP, Yalçın S, Gündüz G, et al. Poly (DL-Lactic-Co-Glycolic Acid) MicroparticleDoxorubicin Formulations for Anti-cancer Drug Delivery. IUFS Journal of Biology. 2014;73(1):9-19. doi: 10.18478/IUFSJB.31212.

58. Costa M, Feitosa A, Oliveira F, et al. Controlled Release of Nor- $\beta$-lapachone by PLGA Microparticles: A Strategy for Improving Cytotoxicity against Prostate Cancer Cells. Molecules. 2016;21(7):873-873. doi: 10.3390/molecules21070873.

59. He Y, Park K. Effects of the Microparticle Shape on Cellular Uptake. Molecular pharmaceutics. 2016 03/01;13(7):2164-2171. doi: 10.1021/acs.molpharmaceut.5b00992. PubMed PMID: PMC5315697. 